\title{
Clinician's awareness and knowledge on the management of differentiated thyroid cancer and the use of radioactive iodine in Tanzania
}

\author{
LULU SAKAFU ${ }^{1 *}$, TEDDY MSELLE ${ }^{2}$, JULIUS MWAISELAGE ${ }^{1}$, AMINA MSENGWA $^{3}$, KHAMZA MAUNDA ${ }^{1}$ and \\ BOUYOUCEF S. EDDIN4 \\ 'Department of Clinical Oncology, Muhimbili University of Health and Allied Sciences, P.O. Box 65001, Dar es \\ Salaam, Tanzania \\ ${ }^{2}$ Department of Biochemistry, Muhimbili University of Health and Allied Sciences, Dar es Salaam, Tanzania \\ ${ }^{3}$ Department of Statistics, University of Dar es Salaam, Dar es Salaam, Tanzania \\ ${ }^{4}$ Department of Nuclear Medicine, CHU Bab El Oued University Algiers, Algeria
}

\begin{abstract}
Introduction: Thyroid cancer is the most common endocrine malignancy worldwide with better outcome if timely and properly managed. Surgery followed by radioactive iodine (RAI) ablation remains the mainstay in the management of differentiated thyroid cancer (DTC). In Tanzania however, few patients with DTC receive RAI ablation post-surgery, and most of these present late, with advanced disease resulting in poor treatment outcome. The objective of this study was to determine the cause for late referral by assessing clinician's awareness and knowledge on the management of DTC and the use of RAI in Tanzania.

Methods: This descriptive cross sectional study was carried out in referral hospitals in Tanzania. Data collection used a standardized self-administered questionnaire. Information sought included awareness, knowledge and practices of clinicians on the management and the use of RAI on patients with DTC was explored.

Results: Majority of clinicians managing DTC patients were males (86\%), in-training surgeons (54\%), and attended less than ten DTC patients per year (62\%). About $44 \%$ of in-training surgeons never heard of nuclear medicine $(p=0.04)$, and $20 \%$ were not aware of the role of RAI in the management of DTC $(p=0.031)$. Only $7.3 \%$ of surgeons were aware of the role of thyroxine therapy, or RAI ablation post-surgery. Of all DTC patients managed surgically, only $16 \%$ had total thyroidectomy with lymphadenectomy $(p=0.05)$.

Conclusion: The findings of this study indicate there is insufficient knowledge on proper management and use of RAI on patients with DTC among clinicians in Tanzania.

Keywords: Differentiated, thyroid, cancer, radioiodine, clinicians, Tanzania
\end{abstract}

\section{Introduction}

Thyroid cancer is the commonest endocrine malignancy worldwide and accounts for about $1 \%$ of all cancers in the world (Globocan, 2010; Brown et al., 2011). Although thyroid cancer is relatively rare, a number of countries have reported the condition to be prevalent in some African countries (Ogbera \& Kuku, 2011). In Tanzania, thyroid cancer accounts for $1.2 \%$ of all cancers attended at the Ocean Road Cancer Institute in Dar es Salaam (Mwaiselage et al., 2013). Histologically, the two most frequent subtypes of thyroid cancer are papillary and follicular cancers (Akslen \& LiVolsi, 2000; Mazzaferri \& Massoll, 2002; Passler \& Scheuba, 2004; Brown et al., 2004). About $90 \%$ of thyroid cancers worldwide are differentiated and these include follicular and papillary cancers (Mazzaferri, 1999). Clinically, most differentiated thyroid cancers (DTC) are asymptomatic (Manel et al., 2010) and hence histological, radiological and laboratory investigations are required to confirm the diagnosis, stage the patient and plan initial management (Wanebo et al., 1983; Hindié et al., 2007; Blesa et al., 2010).

Surgery is the mainstay of the management of DTC, where total or near total

\footnotetext{
*Correspondence E-mail:willymeena2007@yahoo.com
} 
thyroidectomy with lymphadenoctomy is done especially to high risk patients (Rahman, 2011).Three to four weeks post-surgery, intermediate and high risk patients require radioactive iodine (RAI) ablation to remove any residual, gross or microscopic tumor and these patients require thyroxine hormone supplementation for life. The thyroxine supplementation is important to ameliorate the hypothyroid state caused by surgery and to suppress the recurrence rate (Mazzaferri \& Kloos, 2001).

Our observation at the Ocean Road Cancer Institute (ORCI) Thyroid Clinic has revealed three major challenges in patients referred for RAl. These are (i) late referral for RAl ablation; (ii) lack of thyroxine replacement therapy in the interim; and (ii) residual thyroid tissue. Patients with residual thyroid tissue have to be referred back for completion of thyroidectomy, which further delays RAI ablation, and may be associated with poor treatment outcomes ( Hindié et al., 2007). This picture could be attributed by overall lack of knowledge on thyroid cancer management among clinicians in developing countries (Amgad et al., 2012). The objective of this study therefore, was to determine clinicians' knowledge on staging and approach to management of patients with thyroid cancer in Tanzanian referral hospitals.

\section{Materials and Methods}

\section{Study sites}

This was a 5-month hospital based descriptive cross sectional study carried out between December 2014 and April 2015, at the Muhimbili National Hospital (MNH), Bugando Medical Centre (BMC), Kilimanjaro Christian Medical Centre (KCMC), and Mbeya Zonal Referral Hospital (MZRH). MNH is a National Referral and Teaching Hospital located in Dar es Salaam. It has a 1,500 bed facility, attending 1,000 to 1,200 outpatients per day and admitting 1,000 to 1,200 inpatients per week. BMC is a consultant and teaching hospital located in Mwanza and serves the Lake and Western zones of Tanzania (which include regions of Mwanza, Mara, Kagera, Shinyanga, Tabora and Kigoma). It has 900 beds and about 900 employees. KCMC is a referral and teaching hospital with about 800 bed capacity located in Moshi in northern Tanzania. It serves the population of Kilimanjaro, Arusha, Manyara and Tanga regions. MZRH is a 477 bed capacity tertiary hospital located in Mbeya in the southern highland zone. It serves the populations of Ruvuma, Rukwa, Iringa and Mbeya regions. All the four hospitals perform thyroid surgery before referring patients with histologically proven DTC to Ocean Road Cancer Institute. At the time of this study, ORCI was the only facility in the country with radiotherapy and radioiodine therapy capability.

\section{Data collection}

Using a self-administered structured questionnaire, awareness, knowledge and practices of clinicians managing DTC patients in the four hospitals were assessed. A convenient sampling was used to recruit a total of 50 consented clinicians involved in the management of patients with DTC.

\section{Data analysis}

Data were analyzed using Statistical Packages for Social Science (SPSS) version 20. During further analysis residents and registrars were grouped as in-training surgeons due to their expertise and similarity in the work they perform. In subsequent analysis, zonal hospitals (KCMC, MRZH and $B M C$ ) were combined as one group, and then compared to the national referral hospital ( $M N H)$ because it has more financial and human resource.

\section{Ethical considerations}

Study approval was obtained from the Muhimbili University of Health and Allied Sciences review board. The permission to conduct the study obtained from the Authorities of the respective 
referral hospitals.

Results

\section{Baseline characteristics of the study population}

A total of 50 clinicians involved in the management of thyroid cancer patients in the four referral hospitals in Tanzania were recruited. The majority of clinicians were males, with a male to female ratio of 6:1. There were a total of $23(46 \%)$ surgeons, and $27(54 \%)$ in-training surgeons. Majority of participants (56\%) were from Muhimbili National Hospital (Table 1 ).

Table 1: Sociodemographic distribution of study participants $(\mathrm{N}=50)$

\begin{tabular}{lcc}
\hline Characteristics & $\mathbf{N}$ & $(\%)$ \\
\hline Sex & & \\
$\quad$ Male & 43 & $86 \%$ \\
$\quad$ Female & 7 & $14 \%$ \\
Position & & \\
$\quad$ Surgeon & 23 & $46 \%$ \\
$\quad$ In-training surgeons & 27 & $54 \%$ \\
Institution & & \\
$\quad$ Muhimbili National Hospital & 28 & $56 \%$ \\
$\quad$ Kilimanjaro Christian Medical Centre & 7 & $14 \%$ \\
Mbeya Zonal Referral Hospital & 3 & $6 \%$ \\
$\quad$ Bugando Medical Centre & 12 & $24 \%$ \\
\hline
\end{tabular}

\section{Knowledge on differentiated thyroid cancer management}

Most surgeons taking care of DTC were from MNH 17 (73\%). Other hospitals accounted for only $27 \%$ of the surgeons. This difference was statistically significant $(p=0.019)$ (Table 2$)$. On average, each MNH clinician attended fewer than 10 DTC patients per year. However, clinicians from the zonal hospitals attended more than 10 patients per year, and this difference was statistically significant $(p=0.003)$. The surgical procedures frequently carried out on DTC patients were lobectomy, near total or total thyroidectomy. Only $8(16 \%)$ were total thyroidectomy with lymphadenectomy $(p=0.05)$. More than $90 \%$ of clinicians knew that RAI has a role to play in management of DTC post-surgery $(p=0.031)$.

Table 2: Management of differentiated thyroid cancer and role of radioiodine

\begin{tabular}{|c|c|c|c|c|c|}
\hline Variable & & MNH & Zonal Hospitals & Total & P-value \\
\hline \multirow[t]{2}{*}{ Clinicians } & Surgeons & 17 & 6 & 23 & \\
\hline & $\begin{array}{l}\text { In-training } \\
\text { surgeons }\end{array}$ & 11 & 16 & 27 & 0.019 \\
\hline \multirow{2}{*}{$\begin{array}{l}\text { No. of DTC patients } \\
\text { per year }\end{array}$} & $>10$ & 12 & 19 & 31 & \\
\hline & $<10$ & 16 & 3 & 19 & 0.003 \\
\hline \multirow[t]{2}{*}{ Type of surgery } & $\begin{array}{l}\text { Lobectomy or } \\
\text { near total } \\
\text { thyroidectomy }\end{array}$ & 25 & 16 & 41 & \\
\hline & $\begin{array}{l}\text { Total } \\
\text { thyroidectomy } \\
\text { with } \\
\text { lymphadectomy }\end{array}$ & 3 & 6 & 9 & 0.051 \\
\hline \multirow{2}{*}{$\begin{array}{l}\text { Role of RAI in DTC } \\
\text { Management }\end{array}$} & Yes & 28 & 17 & 45 & \\
\hline & No & 0 & 5 & 5 & 0.031 \\
\hline
\end{tabular}




\section{Clinician's knowledge on staging before surgery}

We explored clinician's awareness and knowledge on the type of investigations that are required to help in the staging and management of DTC patients. The modality of investigations used varied among cadres with histology being frequently requested by in-training surgeons whereas, more surgeons opted for radiological (imaging), surgical and clinical staging (Figure 1). Surgeons had overall better knowledge on investigation modalities required for DTC patients compared to in-training surgeons except in histological staging, however the difference was not statistically significant.

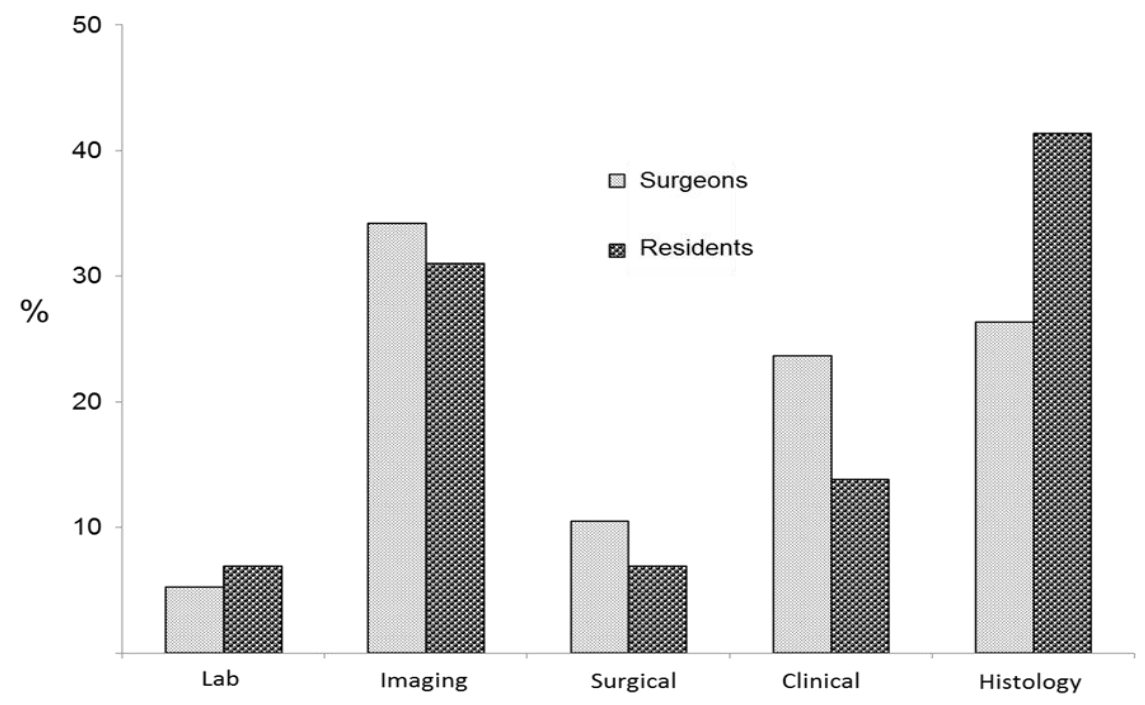

Figure 1. Clinician's knowledge on staging investigations

\section{Clinicians approach to DTC patients following diagnosis}

We determined what clinicians do with patients after a diagnosis of DTC is made. Surgery was the mainstay mode of treatment (62-85\%) among clinician's in all four hospitals. However, only about one-fifth of the surgeons referred patients for further management which included RAl, postsurgery. Only $7.3 \%$ were aware of the role of medical therapy (Figure 2 ).

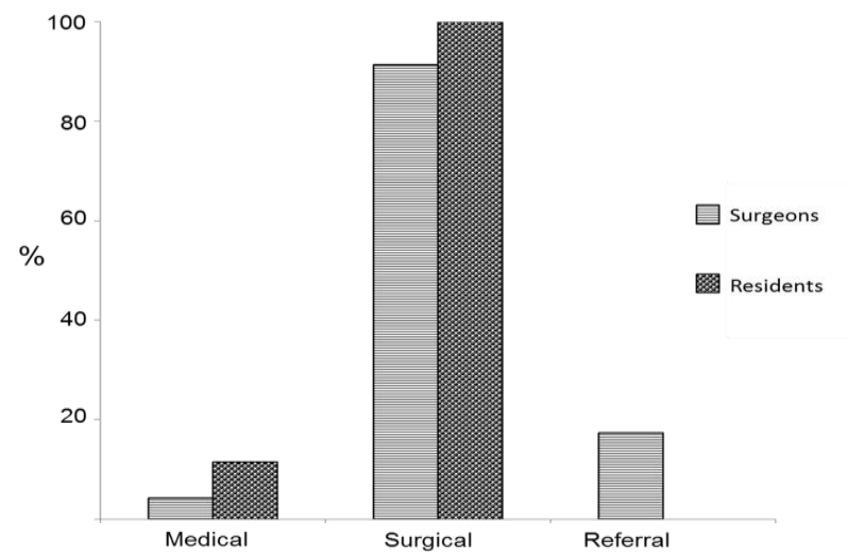

Figure 2: The proportion of surgeons and residents performing DTC

\section{Knowledge of post-surgery management of DTC}

We investigated clinician's knowledge on what else should be done to patients with DTC after surgery. Overall, clinicians were aware of the role of RAI, and RAI was listed by most clinicians 
(78\%) as the first line of management of DTC post-surgery in all four hospitals (Figure 3). However, external beam radiation therapy (EBRT) and chemotherapy which are not routinely indicated in the management of DTC ranked higher than thyroxine replacement (medical). Only (7\%) of clinicians thought there was a need for medical (thyroxine) replacement post-surgery.

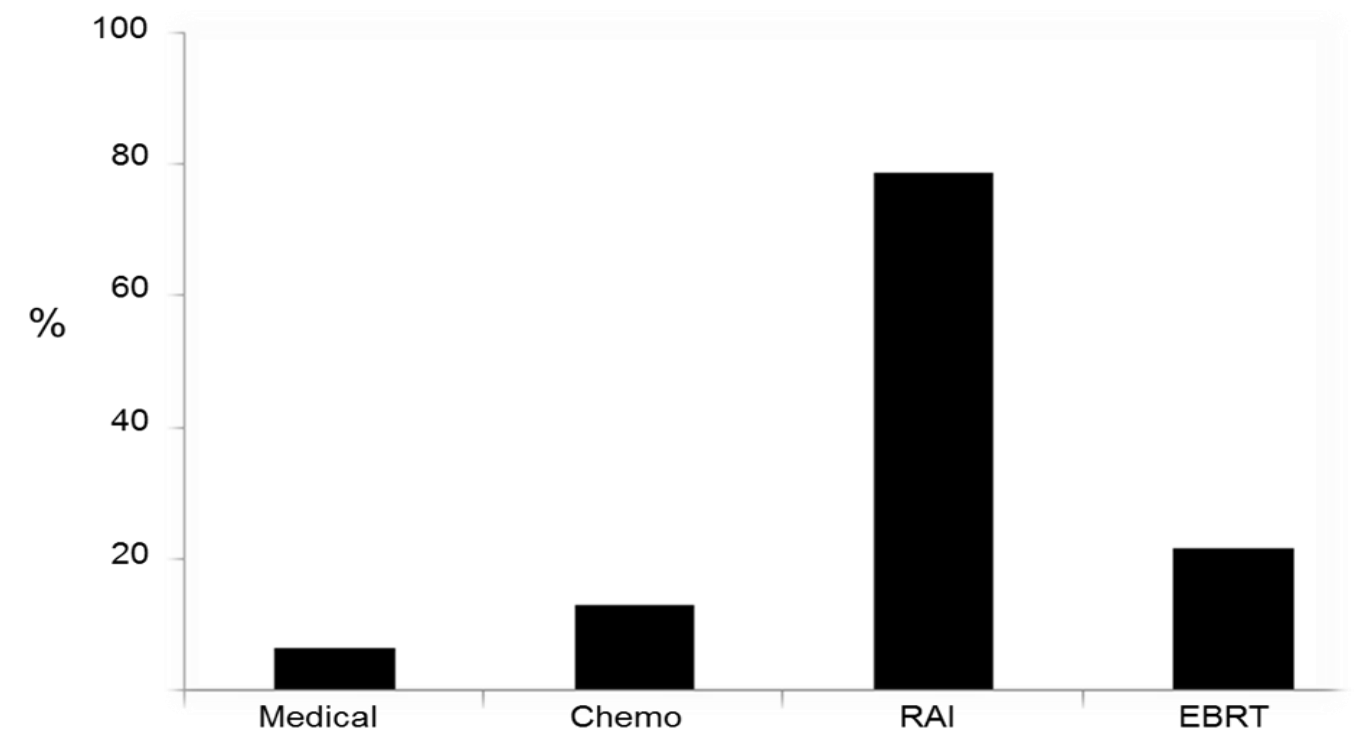

Figure 3. Post-surgery management of DTC

\section{Discussion}

Differentiated thyroid cancer patients have higher probability of complete recovery from the disease if detected early followed by prompt and proper management. However, this may not be the case in Tanzania where patients are diagnosed at referral hospitals, and only a few are referred to Ocean Road Cancer Institute $(\mathrm{ORCl})$ for radioactive iodine therapy (RAI). This is further complicated by the fact that even those referred for RAI, majority come either too late or with extensive residual disease necessitating back referral to the surgeon for completion thyroidectomy.

The findings of this study indicate misdistribution of care providers where most surgeons are at the Muhimbili National Hospital while in the zonal hospitals, majority of clinicians managing DTC patients are in-training surgeons. Inadequate human resource in Tanzania is prevalent in almost all professional cadres. In addition, there is imbalance in the distribution of health workers, with the rural areas suffering most (Munga \& Mæstad, 2009). In a recent analysis, it was shown that although the health workforce situation is slowly improving but shortages of health workers continue to exist throughout the country. By 2013, there were 5.4 doctors and nurse/midwives per 10,000 populations (Mboera et al., 2015).

Patients with DTC require laboratory investigations including histology to confirm diagnosis of malignancy and enable staging before surgery. This is because $8-33 \%$ of follicular thyroid cancer patients may have distant metastasis at diagnosis (Hindié et al., 2007), which is associated with poor patient survival and decreases 5 years survival by 50\% (Hindié et al., 2007; Muresan et al., 2008). Distant metastases are best detected by radiological studies including nuclear medicine studies (Rosenthal, 1997). However, our results indicate that in Tanzania, these radiological studies are infrequently ordered by clinicians across all cadres. Apart from distance metastasis detection, the proper management of thyroid cancer requires information on stage, tumor size and lymph node involvement. This information can best be determined during surgery 
(Rahman, 2011). In this study we found that surgical staging was rarely done, which may lead to improper management because the extent of the disease could not be properly appreciated.

The lack of knowledge on detection of distance metastasis, tumor size and lymph node involvement can be accounted by overall lack of knowledge on cancer management among clinicians in developing countries (Amgad et al., 2012). This oversight may lead to poor surgical management resulting in inadequate surgery and significant residual tissue necessitating repeat completion thyroidectomy (Shah et al., 2003). Completion thyroidectomy however, delays RAI ablation, exposes patients to second surgical stress, and may contribute to poor patient outcome. Studies elsewhere have shown that the more extensive the surgery especially to intermediate and high risk patients, the better is the prognosis (Shah et al., 2003). Further, because surgery is an art, studies have revealed that the higher the number of patients a surgeon attends per year, the more experience he acquires which leads to better patients outcome (Rahman, 2011). This study shows that in referral hospitals of Tanzania on average a clinician attends to less than 10 DTC patients per year. This low numbers could be due to lack of specialized DTC clinics and lack of surgeons specialized in thyroid surgery taking in consideration its low prevalence rate. As a result, any general surgeon, and in some cases in-training surgeons can perform the surgery which may lead to inadequate surgery.

The recommended surgical procedure for intermediate and high risk DTC patients is total thyroidectomy with adapted lymphadenectomy which has been shown to increase survival in this group of patients (Cooper et al., 2009; Shah et al., 2003). In this study however, the commonest surgical procedure was lobectomy or near total thyroidectomy, which could be accounted by the lack of knowledge on risk stratification or local guidelines on thyroid cancer surgery.

Replacement thyroxine therapy is mandated for all thyroid cancer patients post-surgery, to address the lack of intrinsic thyroxine and avoid development of hypothyroidism (Mazzaferri \& Kloos, 2001). Thyroxine supplementation blocks thyrotopin secretion which would otherwise promote DTC regrowth and recurrence, consequently increasing patient survival (Barbaro et al., 2003; Pacini et al., 2006). This study however revealed that many clinicians are not aware of the role of medical treatment which includes thyroxine supplementation post-surgery. It was also surprising to find out that some of the clinicians did not refer patients for RAl therapy at ORCI even though they admitted to be aware of its importance in the management of DTC. This could be attributed to lack of knowledge regarding the availability of RAI at ORCI and also lack of local protocols and management guidelines on thyroid cancer.

Much as studies have shown that DTC responds poorly to chemotherapy and EBRT and that these are only reserved for advanced tumors which are unresectable and unresponsive to RAI (O'Neill et al., 2010), this study has shown that some clinicians still referred patients to ORCI for EBRT and chemotherapy. This not only reflects lack of knowledge in thyroid cancer management, but also lack of local protocols and guidelines. Similar findings have been reported elsewhere (Amgad et al., 2012). Furthermore, until the present time, the medical curricula in Tanzania do include nuclear medicine as part of courses taught at medical schools. This could account for the lack of knowledge on the proper management of thyroid cancer and the use of RAl among clinicians.

In conclusion, this study demonstrates the insufficient knowledge on proper management, and use of RAI in patients with DTC among clinicians in Tanzania, thus increase of nuclear medicine awareness among medical doctors, continuing medical education to practicing clinicians and development of national guidelines for the management of thyroid cancer in Tanzania are required

\section{Competing interests}

Authors declare that there are no competing interests. 


\section{Acknowledgements}

This study was made possible by financial support from the Tanzania Commission for Science and Technology.

\section{Author's contributions}

LS designed the study, collected the data, made the analysis, wrote the manuscript. TM supervised study designing, data collection, data analysis and manuscript writing. AM, JM assisted in study design, data analysis and critical review. KM, BS supervised study designing, data collection, data analysis and manuscript writing. All read and approved the final draft of the manuscript.

\section{References}

Akslen, L. \& LiVolsi, V. (2000) Prognostic significance of histologic grading compared with sub classification of papillary thyroid carcinoma. Cancer 88, 1902-1908.

Amgad, M., Shash, E. \& Gaafar, R. (2012) Cancer education for medical students in developing countries: where do we stand and how to improve? Critical Review of Oncology/Hematology 84, 122-129.

Barbaro, D., Boni, G., Meucci, G., Simi, U., Lapi, P., Orsini, P., Pasquini, C., Piazza, F., Caciagli, M. \& Mariani, G. (2003) Radioiodine treatment with $30 \mathrm{mCi}$ after recombinant human thyrotropin stimulation in thyroid cancer: effectiveness for postsurgical remnants ablation and possible role of iodine contents in L-Thyroxine in the outcome of ablation. Journal of Clinical Endocrinology and Metabolism 88, 4110-4115.

Blesa, M.J.G., Pulido, E.G., Pulla, M.P., Candel, V.A., Canales, J.B.L., Arrom, M.G. \& Rico, P.M. (2010) Old and new insights in the treatment of thyroid carcinoma. Journal of Thyroid Research Article ID 279468.

Brown, R.L., de Souza, J.A. \& Cohen, E.E.W. (2011) Thyroid cancer: burden of illness and management of disease. Journal of Cancer 2, 193-199.

Cooper, D.S., Doherty, G.M., Haugen, B.R., Kloos, R.T., Lee, S.L., Mandel, S.J., Mazzaferri, E.L., Mclver, B., Pacini, F., Schlumberger, M., Sherman, S.I., Steward, D.L. \& Tuttle, R.M. (2009) Revised American Thyroid Association management guidelines for patients with thyroid nodules and differentiated thyroid cancer. Thyroid 19, 1167-1214.

Globocan (2010) Estimated cancer incidence, mortality, prevalence and disability-adjusted life years (DALYs) worldwide in 2008. International Agency for Research on Cancer. World Health Organization. Available at: http://globocan.iarc.fr/

Hindié, E., Zanotti-Fregonara, P., Keller, I., Duron, F., Devaux, J.Y., Calzada-Nocaudie, M., Sarfati, E., Moretti, J.L., Bouchard, P. \& Toubert, M.E. (2007) Bone metastases of differentiated thyroid cancer: impact of early $131 \mathrm{I}$-based detection on outcome. Endocrine-Related Cancer 14, 799-807.

Mazzaferri, E.L. (1999) An overview of the management of papillary and follicular thyroid carcinoma. Thyroid 9, 421-427.

Mazzaferri, E.L. \& Kloos, R.T. (2001) Current approaches to primary therapy for papillary and follicular thyroid cancer. Journal of Clinical Endocrinology and Metabolism 86, 1447-1463.

Mazzaferri, E.L. \& Massoll, N. (2002) Management of papillary and follicular (differentiated) thyroid cancer: new paradigms using recombinant human thyrotropin. Endocrine-Related Cancer 9, 227-247. 
Mboera, L.E.G., Ipuge, Y., Kumalija, J. Rubona, C.J., Perera, S., Masanja, H. \& Boerma, T. (2015) Mid-term review of national health plans: an example from the United Republic of Tanzania. Bulletin of the World Health Organization 93: 271-278.

Munga, M.A. \& Mæstad, O. (2009) Measuring inequalities in the distribution of health workers: the case of Tanzania. BMC Human Resources for Health 7:4.

Muresan, M.M., Olivier, P., Leclere, J., Sirveaux, F., Brunaud, L., Klein, M., Zarnegar, R. \& Weryha, G. (2008) Bone metastases of differentiated thyroid carcinoma. Endocrine-Related Cancer 15, 37-49.

Mwaiselage, J., Ngoma, T., Yiannoustsos, C., Wools-Kaloustian, K., Somi, G., Juma, J. Cancer incidence in Dar es Salaam, Tanzania: 2007 - 2010. Proceedings of the UICC World Cancer Congress: Connecting for Global Impact. Montreal, Canada. August 27 - 30, 2012.

Ogbera, A.O. \& Kuku, S.F. (2011) Epidemiology of thyroid diseases in Africa. Indian Journal of Endocrinology and Metabolism 15(Suppl2): S82-S88

O'Neill, C.J., Oucharek, J., Learoyd, D. \& Sidhu, S.B. (2010) Standard and emerging therapies for metastatic differentiate thyroid cancer. Oncologist 15, 146-156.

Pacini, F., Schlumberger, M., Dralle, H., Elisei, R., Smit, J.W., Wiersinga, W., European Thyroid Cancer Taskforce (2006) European consensus for the management of patients with differentiated thyroid carcinoma of the follicular epithelium. European Journal of Endocrinology 154, 787-803.

Passler, C. \& Scheuba, C. (2004) Prognostic factors of papillary and follicular thyroid cancer: differences in an iodine-replete endemic goiter region. Endocrine-Related Cancer 11, 131-139.

Rahman, G.A. (2011) Extent of surgery for differentiated thyroid cancer: recommended guideline. Oman Medical Journal 26, 56-58.

Rosenthal, D.I. (1997) Radiologic diagnosis of bone metastases. Cancer 80, 1595-1607.

Shah, M.D., Hall, F.T., Eski, S.J., Witterick, I.J., Walfish, P.G. \& Freeman, J.L. (2003) Clinical course of thyroid carcinoma after neck dissection. Laryngoscope 113, 2102-2107. 